\title{
1 A connectome-based neuromarker of the non-verbal number acuity and arithmetic skills
}

$6 \quad{ }^{1)}$ Beijing Key Laboratory of Applied Experimental Psychology, School of Psychology, Beijing Normal

7 University, Beijing, China

$8 \quad{ }^{2)}$ Guangdong Key Laboratory for Biomedical Measurements and Ultrasound Imaging, School of Biomedical

9 Engineering, Shenzhen University, Shenzhen, China

$10 \quad{ }^{3)}$ Department of Psychology \& Tsinghua Laboratory of Brain and Intelligence, Tsinghua University, Beijing, 11 China

$15 *$ Correspondence to:

16 Ke Zhou, Ph.D

17 Beijing Key Laboratory of Applied Experimental Psychology,

18 School of Psychology, Beijing Normal University

19 No. 19, Xinjiekouwai Street, Haidian District, Beijing 100875, China

20 E-mail: kzhou@bnu.edu.cn

21 ORCID: https://orcid.org/0000-0003-4773-758X

Jia Liu, Ph.D

24 Department of Psychology \& Tsinghua Laboratory of Brain and Intelligence,

25 Tsinghua University

26 No. 30, Shuangqing Rd, Haidian District, Beijing 100084, China

27 E-mail: liujiaTHU@tsinghua.edu.cn

28 ORCID: https://orcid.org/0000-0003-0383-0934 


\section{$1 \quad$ Abstract}

2 The approximate number system (ANS) is vital for survival and reproduction in animals

3 and crucial in constructing abstract mathematical abilities in humans. Most previous

4 neuroimaging studies focused on identifying discrete brain regions responsible for the ANS and

5 characterizing their functions in numerosity perception. However, there lacks a neuromarker to

6 characterize an individual's ANS acuity, especially one based on the whole-brain functional

7 connectivity (FC). Here, we identified a distributed brain network (i.e., numerosity network)

8 using a connectome-based predictive modeling (CPM) analysis on the resting-state functional

9 magnetic resonance imaging (rs-fMRI) data based on a large sample size. The summed strength

10 of all FCs within the numerosity network could reliably predict individual differences of the

11 ANS acuity in behavior. Furthermore, in an independent dataset from the Human Connectome

12 Project (HCP), we found that the summed FC strength within the numerosity network could also

13 predict individual differences in arithmetic skills. Our findings illustrate that the numerosity

14 network we identified could be an applicable neuromarker of the non-verbal number acuity and

15 might serve as the neural basis underlying the known link between the non-verbal number acuity

16 and mathematical abilities.

20 Keywords: Approximate number system, Numerosity, Connectome-based predictive

21 modeling, Arithmetic skills, individual differences 


\section{Introduction}

2 The number of items in a set is represented in humans by two different representational

3 systems: a language-dependent cultural system that encodes the precise cardinality of elements ${ }^{1}$;

4 and an approximate number system (ANS) shared by adults ${ }^{2,3}$, infants ${ }^{4}$, many animal species ${ }^{5}$,

56,7 , and even deep convolutional neural networks ${ }^{8,9,10}$, which encodes quantity in an

6 approximate, non-symbolic manner without verbally counting (i.e., numerosity perception). In

7 animals, the ANS is of evolutionary importance as it can guide behaviors necessary for survival

8 and reproduction, such as determining the relative amount of food during foraging ${ }^{11,12}$. In

9 humans, the ANS is hypothesized to be the foundation of constructing more abstract

10 mathematical abilities ${ }^{13}$. For example, Harvey et al. ${ }^{14}$ revealed a positive correlation between

11 ANS acuity and math achievement, indicating the importance of ANS in shaping symbolic math 12 skills.

13 Over decades, significant progress has been made to understand how numerosity

14 information is represented in the brain. For instance, neurophysiological studies revealed that

15 neurons in the prefrontal and parietal cortices of macaques were tuned to the numerosity of

16 spatial arrays $15,16,17,18$. In humans, using the fMRI approach, researchers have also found that

17 the numerosity information was mainly represented in the frontoparietal association cortex,

18 including the horizontal part of the intraparietal sulcus, which exhibited similar tuning to

19 numerosity 19, 20, 21, 22 (add He et al., PNAS,2015). However, recent new evidence showed that

20 other brain areas were also involved in processing numerosity information. For example, Harvey

$21{ }^{23}$ has proposed a numerical neural system including visual cortex, inferior temporal gyrus (ITG),

22 fusiform gyrus, and angular gyrus, in addition to the parietal lobe and prefrontal cortex. The

23 earliest numerosity-related neural activity has also been found in the visual cortex, such as V2 
1 and V3 ${ }^{24,25}$, and numerosity information could be decoded in both the early visual and intra-

2 parietal cortex ${ }^{26}$. Number neurons were also found in the medial temporal lobe (MTL) of human

3 neurosurgical patients when they performed calculation tasks on symbolic and non-symbolic

4 stimuli ${ }^{27}$. The angular gyrus also plays a central role in the linguistic number representation

5 system ${ }^{28,29}$. Taken together, the neural representation of the ANS system seems to involve

6 multiple cortical areas. However, most of these previous studies focused on identifying the

7 function of specific brain regions supporting the ANS. There still lacks a connectome-wise

8 neuromarker based on whole-brain functional connectivity (FC) that can characterize an

9 individual's ANS acuity.

10 FC measures the activity synchronization or interaction between fMRI bold oxygen level-

11 dependent (BOLD) signals of two brain areas ${ }^{30,31}$. Whole-brain FC analysis based on network

12 theory provides information on how distributed brain regions simultaneously exchange or

13 integrate information to support specific cognitive functions ${ }^{32}$. Recently, a connectome-based

14 modeling (CPM) analysis ${ }^{33}$ has been proposed to predict individual differences in human

15 behavior (e.g., cognitive abilities) based on participants' whole-brain FC pattern. The CPM

16 analysis provides a complementary measure of human cognitive abilities to traditional

17 approaches that focused on specific functions of discrete brain regions. The CPM approach can

18 be successfully applied to predict individual differences in several cognitive abilities, such as

19 fluid intelligence $(\mathrm{gF})^{34}$, sustained attention ${ }^{35}$, reading accuracy ${ }^{36}$, and creative ability ${ }^{37}$.

20 Because substantial variations also exist in the ANS acuity among individuals ${ }^{14,38,39}$, here in the

21 present study, we aimed to employ the CPM analysis to develop a whole-brain neuromarker (i.e.,

22 numerosity network) for the ANS acuity based on the resting-state fMRI data. 
In addition, previous studies also illustrated that the CPM identified with one task could be generalized to predict the performance of other related tasks. For instance, sustained attention

3 CPM, derived from healthy individuals in a sustained attention task, could also predict the

4 ADHD-Rating-Scale (ADHD-RS) score in the ADHD patients ${ }^{35}$, and the reading recall accuracy

5 in another group of healthy individuals ${ }^{36}$. As mentioned above, because the ANS plays a crucial

6 role in determining an individual's math achievement ${ }^{14}$, the second aim of the present study is to

7 examine whether the numerosity network that we identified above could also predict an

8 individual's arithmetic skills.

11 constructed a numerosity CPM based on their resting-state fMRI data to predict the individual

12 differences in ANS acuity, using a leave-one-out (LOO) cross-validation procedure. As a result,

13 the summed FC strength within the numerosity network could predict individual differences of

14 the ANS in the left-out participant. More importantly, we further examined whether the

15 numerosity network can predict the performance of arithmetic skills in an independent dataset

16 from the Human Connectome Project (HCP). Our results suggested that the summed FC strength

17 within the numerosity network could also predict individual differences in arithmetic skills, not

18 language comprehension abilities. In sum, our findings demonstrate that the ANS acuity can be

19 reliably predicted from the strength of the numerosity network of each individual, and imply that

20 the numerosity network might also serve as the neural basis underlying the known link between

21 ANS acuity and mathematical ability.

\section{Results}




\section{Behavioral performance}

The present investigation included an ANS dataset and an HCP math/story dataset. The

3 ANS dataset consisted of a primary dataset and a validating dataset. It was used to obtain and

4 verify the numerosity network. The HCP math/story dataset was used to investigate whether the

5 numerosity network identified in the previous ANS dataset could predict individual differences

6 in arithmetic skills and/or language comprehension abilities. In the ANS dataset, we calculated

7 the Weber fraction for each participant, representing the participant's ANS acuity ${ }^{14}$. In the HCP

8 math/story dataset, we calculated two measures of the inverse efficiency score (IES) for each

9 participant, representing the participant's arithmetic skills and language comprehension abilities,

10 respectively ${ }^{40}$. Larger scores of Weber fraction or IES performance indicate poorer numerosity

11 acuity or arithmetic skills, respectively. The mean performance for these behavioral tasks of all

12 datasets was shown in Table 1.

13 Because the Jarque-Bera tests of normality revealed that these behavioral scores were not

14 normally distributed (Fig. 1 B-E), we thus used Spearman's rank correlation for the edge

15 selection in establishing the numerosity network during the CPM analysis. Note that the

16 numerosity network was constructed using the ANS primary dataset and validated using the

17 ANS validating dataset.

18 Numerosity network can predict the individual differences in the ANS acuity

19 In the ANS primary dataset (141 participants), using the Shen et al. protocol ${ }^{33}$, we adopted

20 the LOO cross-validation method to test whether the intrinsic FC profile can predict the Weber

21 fraction. For each LOO iteration, all 141 participants were divided into training (140

22 participants) and testing (1 left-out participant) sets. In the training set, for each edge between

23 pair of nodes within the Shen's atlas ${ }^{41}$, we calculated the correlation between its FC and the 
1 Weber fraction across participants. Edges showing significant positive or negative correlation

$2(p<0.01)$ composed a positive or negative predictive network, respectively. Next, for each of the

3140 participants, we calculated the summed strength of all FCs within the positive and negative

4 networks separately. We constructed a general linear model (GLM) for both the positive and

5 negative networks by relating the summed strength to the Weber fraction across 140 participants

6 in the training set. Then, in the testing set, the GLM was used to obtain the predicted Weber

7 fraction of the left-out participant from his/her summed FC strength within the positive and

8 negative network separately.

Across all 141 LOO iterations, we then calculated the Pearson's correlation between the

10 observed and predicted Weber fraction scores to evaluate the predictive power. For the positive

11 networks, the correlation between observed and predicted Weber fractions was significant $(r=$

$120.204, p=0.015)$, while for the negative networks, the correlation was non-significant $(r=$

$130.032, p=0.703$ ) (Fig. 2). We carried out a permutation test to further confirm the reliability of

14 these results. We shuffled the behavior performance across participants 10,000 times and

15 calculated the Pearson's correlation coefficients between observed (randomly shuffled) and

16 predicted Weber fraction. We found that the positive network outperformed the set of 10,000

17 permutation tests $\left(p_{\text {perm }}=0.0081\right)$. In comparison, the negative network did not outperform the

18 set of 10,000 permutation tests $\left(p_{\text {perm }}=0.3510\right)$. There was a significant difference in the

19 predictive power between the positive and negative networks (Steiger's $\left.z=2.67, p=7.6 \times 10^{-3}\right)^{42}$.

20 It thus suggests that the strength of the functional connectivity profile within the positive

21 networks could predict individual differences in ANS acuity. However, the negative network did

22 not show the predictive ability of the individual differences in ANS acuity. Therefore, in the

23 subsequent analysis, we focused on the predictive power of the positive network. Note that the 
1 predictive ability of the numerosity network cannot be alternatively explained by head motion, as

2 the average frame-to-frame motion was not correlated with Weber fraction $(r=-0.0149, \mathrm{p}=$

$3 \quad 0.8607)$.

4 Note that the positive networks differed across iterations. Across all 141 iterations, the

5 number of edges within the positive ranged from 114 to 155 . We generated a final numerosity

6 network by selecting the overlapping edges of the positive networks across all iterations. There

$7 \quad$ were 87 nodes and 80 edges in the final numerosity network.

8 Then, in the independent validating dataset (112 participants), we evaluated whether the

9 final numerosity network could successfully predict the individual differences of the ANS acuity.

10 The correlation between the summed strength of the numerosity network and the Weber fraction

11 across participants was significant $(r=0.236, p=0.012)$ (Fig. 3 A). We also conducted the same

12 permutation test to confirm this result further. The numerosity network outperformed the set of

1310,000 permutations where we randomly shuffled the behavior performance across participants

$14\left(p_{\text {perm }}=0.0060\right)$. It thus indicated that the final numerosity network we identified could serve as a

15 connectome-based neuromarker to predict an individual's ANS acuity reliably.

16 Summed FC strength within the numerosity network can predict arithmetic

17 skills

18 To address our second aim, we further evaluated whether the summed FC strengths within

19 the numerosity network could predict the individual differences in the arithmetic skills in an

20 HCP math/story dataset. We found that the summed FC strengths within the numerosity network

21 we identified above correlated significantly with the IESs in the math task $(r=0.247, P=0.006)$

22 (Fig. 3 B). The numerosity network also outperformed the set of 10,000 permutation tests where

23 we randomly shuffled the IES performance in the math task across participants $\left(P_{\text {perm }}=0.0025\right)$. 
1 The greater the summed FC strength within the numerosity network, the larger the IES measure

2 of the arithmetic skills. Greater summed FC strength within the numerosity network pointed to

3 both the higher Weber fraction (i.e., poorer numerosity precision) in the NC task and the larger

4 IES (i.e., poorer arithmetic skills) in the math task. It thus indicates that the numerosity network

5 could also predict the performance of arithmetic skills, even in a completely new dataset.

6 We next evaluated whether the numerosity network could predict the IES of the story task.

7 We found that the summed FC strength within the numerosity network could not predict the IES

8 in the story task $(r=0.109, P=0.234)$ (Fig.3 C). We again ran permutation tests and found that

9 the numerosity network failed to outperform the set of 10,000 permutations where we randomly

10 shuffled the IES performance in the story task across participants $\left(P_{\text {perm }}=0.1161\right)$.

11 In short, these findings suggest that the numerosity network could only predict the

12 individual differences in the arithmetic skills in specific, but not language comprehension

13 abilities in general.

\section{Anatomical locations of network edges}

We also examined the Anatomical locations of network edges within the numerosity

16 network. The numerosity neuromarker involves a distributed neural network in the neocortex

17 (Fig. 4B). As shown in Fig. 4A, in the numerosity network, 45 nodes were in the right

18 hemisphere, and 42 nodes were in the left hemisphere. 18, 17, 13, 13, 12, 11, and 3 nodes located

19 in seven macroscale regions (i.e., the temporal, prefrontal, motor, limbic, occipital, parietal, and

20 insula) in Shen's atlas ${ }^{41}$ (Fig. 4C). Contralateral connections (50 edges) were more common

21 than ipsilateral connections (30 edges) in the numerosity network $\left(\chi^{2}(1)=5.0022, p=0.0253\right)$.

22 There were 19 right-right connections and 11 left-left connections in the numerosity network

$23\left(\chi^{2}(1)=2.3793, p=0.1229\right)$. 
The numerosity networks include many numerosity-related brain regions previously revealed, such as the early visual cortex ${ }^{43}$, parietal sensory cortex ${ }^{26}$, angular gyrus ${ }^{44}$, medial

3 temporal gyrus (MTG) ${ }^{27}$, the motor cortex ${ }^{45}$, and prefrontal cortex ${ }^{18}$. However, some regions in

4 the numerosity network, such as the visual association cortex, inferior temporal gyrus (ITG), and

5 fusiform gyrus, have not been reported to be involved in processing numerosity information. The

6 occipital (primary visual and visual association) nodes mostly connect to parietal and motor

7 regions (Fig. 5A). There are dense connections between left temporal nodes and right motor,

8 right parietal regions (Fig. 5B, 5C). The PFC nodes mainly connect within the lobes (Fig. 5D).

9 Detailed information of all nodes and edges could be found in supplementary materials.

11 according to their degree centrality ${ }^{46}$ (DC; i.e., the number of direct functional connections

12 between a given node and the rest nodes within the numerosity network). The top 27 nodes with

13 high DC are presented in Table 2 (DC $>1)$. Regions with the highest $\mathrm{DC}$ indicates the hub regions

14 of the numerosity network, including brain regions in the occipital (e.g., right primary visual

15 cortex, Brodmann area $(\mathrm{BA}) 17, \mathrm{DC}=11$ ), motor (e.g., right premotor cortex, $\mathrm{BA} 6, \mathrm{DC}=6$ ), and temporal (e.g., left ITG, BA 20, DC = 5) cortex.

\section{Discussion}

In the present study, using the CPM analysis, we obtained a numerosity network based on the numerosity comparison task, whose strength could reliably predict the individual differences

21 in the ANS acuity among individuals. This identified numerosity network consisted of functional connections between distributed brain regions, suggesting that a whole-brain, widely distributed numerosity network could serve as a reliable neuromarker for an individual's non-symbolic 
1 numerosity ability. The robust predictive power of this numerosity neuromarker was further

2 verified in a completely new dataset with the same behavioral task.

3 Only the positive network could predict individual differences in the ANS acuity. The

4 negative network could not reliably predict the ANS acuity among individuals. Similar to our

5 results, in predicting individual gF score ${ }^{34}$, the negative network also showed less accurate

6 predictions than the positive network. When the nodes were restricted within frontoparietal

7 networks, the positive-feature models showed significant prediction results. In contrast, negative-

8 feature models didn't show significant predictive power ${ }^{34}$. The reason for the inability of the

9 negative network to predict the individual differences in the ANS acuity still needs future

10 investigation.

11 Another significance of our study was that the numerosity neuromarker we identified could

12 also specifically predict individual differences in arithmetic skills. In the literature of numerosity

13 perception, many studies have found a positive correlation between numerosity precision and

14 cognitive arithmetic skills ${ }^{14,47}$, suggesting that the number sense may serve as a 'start-up' tool

15 for mathematics acquisition ${ }^{13}$. Our results provided a neural basis for this important link,

16 suggesting that numerosity perception and arithmetic skills may share similar functional

17 networks. On the contrary, this numerosity network could not predict language comprehension

18 abilities, consistent with previous findings that mathematical processing relies on specific brain

19 areas and dissociates from language processing $48,49,50$.

20 The increased strength of the numerosity network was indicative of poor numerosity acuity

21 or arithmetic skills. It seems to be counterintuitive. However, there were two possible

22 explanations for this finding. One possible explanation was that this result might reflect a kind of

23 compensatory mechanism ${ }^{51}$. The participants with poor numerosity acuity need more sensory 
1 representation of the stimulus. Another possible explanation was that the strength of the

2 numerosity network might represent the control ability to inhibit non-numerical magnitude

3 information when extracting numerosity information, which can characterize numerosity

4 information more accurately $52,53,54,55,56$. However, our current findings cannot provide direct

5 evidence to support any of these two accounts. Therefore, further investigations are needed to

6 address this issue.

At the node level, the numerosity network involves multiple distributed regions, such as

8 occipital regions (early visual cortex, visual association), sensorimotor regions (premotor cortex,

9 supplementary motor cortex, primary sensory cortex), temporal regions (ITG, temporopolar area,

10 and fusiform), and prefrontal areas (Frontopolar area and orbitofrontal cortex). Most of these

11 regions have been suggested to involve in numerosity processing. For instance, Previous studies

12 show that numerosity processing involves at least two temporal stages in the visual cortex ${ }^{57}$.

13 Subsequent studies found that the earliest activity likely arises from areas such as V2 and V3 ${ }^{24}$.

14 The sensorimotor cortex is suggested to be involved in a generalized system with quantitative

15 information processing ${ }^{45}$. In monkeys, neurons in the parietal sensorimotor area respond to the

16 number of self-generated motor actions ${ }^{58}$. A link between mathematics acquisition and motor

17 skills has also been reported in children with learning disabilities, showing a correlation between

18 motor skills and proficiency in solving mathematical problems ${ }^{59}$. Both premotor and parietal

19 areas get additional activation during verbal counting of visual and auditory items ${ }^{60}$. Studies in

20 humans ${ }^{61,62}$ indicated parts of the parietal cortex as a core number system that processes

21 symbolic and non-symbolic numerical magnitude. There is also evidence that single-neuron

22 activity from the medial temporal lobe (MTL) encoded numerical information when participants

23 performed calculation tasks on symbolic and non-symbolic stimuli ${ }^{27}$. Neurons in the lateral 
1 prefrontal cortex responded selectively to a specific number of items (i.e., numerosity) in visual

2 multiple-dot displays ${ }^{63}$. Therefore, the numerosity system might rely on the cooperative work of

3 multiple distributed brain regions.

4 At the edge level, in the numerosity network, occipital regions are connected with angular

5 gyrus, ITG, and fusiform gyrus, which could be conceptualized as a communication process

6 between visual input and visual information extraction, such as visual recognition, spatial

7 orientation, semantic representation processing. Moreover, the numerosity network did include

8 many connections between occipital nodes and sensorimotor nodes, indicating the interaction

9 between visual perception, action planning, and execution in the numerosity system. These

10 results show a crucial role of the sensorimotor cortex in the numerosity system and the strong

11 interaction between different perceptual features in the numerosity system ${ }^{45}$. In addition, the

12 numerosity network shows dense connections between left temporal and right premotor, and

13 between left temporal and right parietal sensorimotor regions. These connections may present

14 functional coupling between ventral and dorsal areas of the numerosity system. The numerosity

15 network also shows connections within the prefrontal lobe. A nonverbal quantification system

16 resides in a dedicated parietal-frontal brain network in primates ${ }^{64}$. However, our numerosity

17 network did not contain any parietal-frontal connections. Our findings provided a

18 complementary measure of numerosity perception and arithmetic skills to traditional approaches

19 that focused on the specific function of discrete brain regions.

20 However, it remains an open question whether the numerosity network we identified could

21 predict changes in ANS acuity during development. Previous research has revealed that the

22 precision of numerical acuity is sharped with age and the acquisition of formal mathematical

23 education, which may reflect an ability to focus on numerical information while filtering out 
1 non-numerical information $53,54,55,56,65$. Testing the change of the numerosity network

2 associated with the within-individual changes in numerosity perception and mathematical

3 abilities over years can inform the common and distinct functional architecture of these

4 processes during development. In particular, it can provide insights into how functional brain

5 organization reflects risk for or resilience to impairments such as developmental dyscalculia or

6 mathematical difficulties, potentially informing early treatments or interventions.

$7 \quad$ A limitation of our study is that the ANS acuity and arithmetical skills were only assessed

8 by specific paradigms. And we didn't include the investigation of symbolic number

9 representation. Further research could include various number-related tasks to verify our findings

10 or address the common and distinct functional architecture of these tasks related to number

11 perception and mathematical abilities.

\section{Methods}

\section{Overview}

In the primary ANS dataset, we constructed a numerosity CPM based on their behavior

16 performance in an NC task and their resting-state $\mathrm{AMRI}$ data, to predict the individual differences

17 in the ANS acuity, using a leave-one-out cross-validation procedure. The reliability of the

18 identified numerosity network was verified in an independent validating ANS dataset with the

19 same NC task. Then in the HCP math/story dataset, we examined whether the identified

20 numerosity network could predict the individual differences in the arithmetic skills.

\section{$21 \quad$ ANS dataset}

22 Participants. The behavioral data, and the structural and resting-state fMRI data were collected

23 from two batches of college students (Batch A and Batch B). Data from the Batch A (154 
1 participants; age: $20-25 ;$ mean $=21.66, \mathrm{SD}=1.07 ; 65$ males) was used to construct the numerosity CPM. Data from the Batch B (145 participants; age: 20-25, mean =21.39, SD =

$30.91 ; 36$ males) was used to validate the reliability of the numerosity network. Batch A and

4 Batch B datasets correspond to the ANS primary and validating datasets, respectively. All

5 participants have no history of neurological disorder (e.g., mental retardation, traumatic brain

6 injury) or psychiatric illness. The experimental protocol was approved by the Institutional

7 Review Board of Beijing Normal University. Written informed consent was obtained from all

8 participants before the study.

9 NC task. Similar to previous research ${ }^{14}$, a classical NC paradigm was used to measure

10 participants' ANS acuity. In each trial, a spatially intermixed blue and yellow dot display was

11 presented on a computer screen for $750 \mathrm{~ms}$ (Fig1. A). Participants indicated which color was

12 more numerous by the keypress. The ratio between the two sets of colored dots varied at 11

13 levels, including 12:11, 11:10, 10:9, 9:8, 8:7, 7:6, 6:5, 10:8, 8:6, 9:6, and 12:6. The color of the

14 dots set was counterbalanced across trials. Dot-size of each ratio level was controlled: the

15 average blue dot size variation was equal to the variation range of the average yellow dot size.

16 Participants completed forty test trials after practicing five practice trials.

17 The minimum distinguishable difference between numbers of blue and yellow dots that

18 produces a noticeable response was estimated as each participant's ANS acuity known as the

19 Weber fraction. The Weber fraction of each participant was estimated by a QUEST routine ${ }^{66,67,}$

20 . The QUEST routine provides a given number of sequential trials and updating the probability

21 distribution function (PDF) of Weber fraction based on the participant's response and current

22 PDF, following the Bayes' Rule. After the final trial, the mean value of PDF was recorded as the 
1 participant's Weber fraction and used to represent the participant's ANS acuity. Thus, a greater

2 Weber fraction score corresponds to the poorer ANS acuity.

3 Participants with divergent estimating sequences were excluded by visual inspection.

4 Participants with Weber fraction greater than 0.5 were also excluded from the subsequent

5 analysis (e.g., Weber fraction greater than 0.5 means an inability to distinguish 12 dots from 6

6 dots). In sum, 13 participants in the ANS primary dataset and 25 participants in the ANS

$7 \quad$ validating dataset were excluded from further analysis.

8 MRI data and preprocessing. The structural and resting-state fMRI data were acquired in the

9 same session. The MRI data were acquired from a Siemens 3T Trio scanner (MAGENTOM

10 Trio, a Tim system) with a 12-channel phased-array head coil at the BNU Imaging Center for

11 Brain Research, Beijing, China. T1-weighted structure images were acquired with a

12 magnetization-prepared rapid gradient-echo (MPRAGE) sequence (TR/TE/TI = $2.53 \mathrm{~s} / 3.45$

$13 \mathrm{~ms} / 1.1 \mathrm{sec}, \mathrm{FA}=7$ degrees, voxel size $=1 \times 1 \times 1 \mathrm{~mm}$, slice thickness $=1.33 \mathrm{~mm}$, number of

14 volumes $=128$ ) for each participant. The resting-state data was acquired using a T2*-weighted

15 GRE-EPI sequence with different parameters from task-state fMRI $(\mathrm{TR}=2000 \mathrm{~ms}, \mathrm{TE}=30 \mathrm{~ms}$,

16 flip angle $=90$ degrees, number of slices $=33$, voxel size $=3.125 \times 3.125 \times 3.6 \mathrm{~mm}$ ).

17 Resting-state fMRI images were preprocessed using the FMRI Expert Analysis Tool

18 version 5.98, part of FMRIB's Software Library (www.fmrib.ox.ac.uk/fs1). The first four

19 volumes of each participant were discarded to allow for the stabilization of magnetization. In

20 addition to head-motion correction, brain extraction, spatial smoothing (FWHM $=6 \mathrm{~mm})$, grand-

21 mean intensity normalization, and removing a linear trend, several other preprocessing steps

22 were also used to reduce spurious variance. These steps included using a temporal band-pass

23 filter $(0.01-0.08 \mathrm{~Hz})$ to retain only low-frequency signals, and regression of the time course 
1 obtained from motion correction parameters, the mean signals of the cerebrospinal fluid and

2 white matter, and the first derivatives of these signals. Then, all functional images were aligned

3 to the structural images using FMRIB's linear image registration tools and warped to the

4 MNI152 template using FMRIB's nonlinear image registration tool. Since head motion might

5 confound functional connectivity analyses, in the validating dataset, we excluded eight

6 participants with frame-to-frame head motion estimation greater than $0.15^{34}$. Finally, 141

7 participants were retained in the ANS primary dataset, and 112 participants were retained in the

8 ANS validating dataset. There was no correlation between head motion and Weber fraction in

9 the retained participants of both datasets (the primary dataset: $r=-0.0149, p=0.8607$; the

10 validating dataset: $r=-0.0068, p=0.9433)$.

11 Functional connectivity calculation. FC was calculated between ROIs or “nodes” in Shen's

12 atlas, which maximized the similarity of the time series of the voxels within each node ${ }^{41,69}$. A

13 subset of nodes of the 268-node functional brain atlas was used in our study because some

14 resting-fMRI scans did not cover the full brainstem and cerebellum. We thus focused on nodes in

15 the neocortex by removing all nodes of the cerebellum, brainstem, and subcortex (67 nodes

16 totally). The remained 201 nodes were shown in Fig. S1. The mean time course of each node was

17 extracted as a measure of spontaneous neural activity in that node. Pearson's correlation

18 coefficients $(r)$ were calculated between the time courses of each pair of nodes and normalized

19 using Fisher's $r$-to-Z transformation. Finally, a 201×201 FC matrix was obtained for each

20 participant in the primary dataset and the validating dataset.

\section{HCP dataset}

22 Participants. Data for evaluating the numerosity network's predictive power in predicting the

23 individual differences in mathematical and language comprehension abilities were from the 
1 Human Connectome Project (Q1 and Q2 HCP data releases; 131 participants; age: 22-35; 30 males). Resting-state fMRI data of both left-right (LR) and right-left (RL) phase-encoding runs

3 (HCP filenames: rfMRI_REST1) were included in subsequent analysis. Consistent with the

4 preprocessing procedure of the ANS dataset, we also excluded seven participants with frame-to-

5 frame head motion estimation greater than 0.15 (violated in either of LR or RL phase-encoding

6 runs, HCP: Movement_RelativeRMS_mean).

7 Behavioral Test. In the HCP language math/story task, participants answered math-related and

8 story-related questions after hearing auditory blocks. The HCP language task consists of two

9 runs that each interleaves four blocks of math task and four blocks of story task ${ }^{70}$. The math task

10 engages participants' attention continuously with mental arithmetic. The math task includes

11 auditory trials and requires participants to complete addition and subtraction problems, followed

12 by a 2-alternative forced-choice task. For example, "Four plus twelve minus two plus nine equals

13 twenty-two or twenty-three?" The math task is adaptive to maintain a similar level of difficulty

14 across participants. The story task includes brief and engaging auditory stories (5-9 sentences)

15 adapted from Aesop's fables. For example, after a story about an eagle that saves a man who had

16 done him a favor, participants were asked the question of "That was about revenge or

17 reciprocity?" For both tasks, participants push a button to select either the first or the second

18 choice. Median reaction time and accuracy were recorded for both math and story tasks (HCP

19 math task: Language_Task_Math_Acc and Language_Task_Math_Median_RT, HCP story task:

20 Language_Task_Story_Acc and Language_Task_Story_Median_RT).

21 We calculated the inverse efficiency score (IES) to integrate response time and accuracy ${ }^{40}$.

22 The IES was calculated as median response time divided by accuracy, to evaluate mathematical

23 and language comprehension abilities. There was no correlation between head motion and IES in 
1 the remaining set of $\mathrm{n}=124$ participants for math and story tasks (LR: IES of Math $\mathrm{r}=0.05, \mathrm{p}=$ 0.5588, IES of Story $r=-0.03, p=0.76$; RL: IES of Math $r=0.01, p=0.9114$, IES of Story $r=-$

$30.09, \mathrm{p}=0.32)$.

4 MRI data and preprocessing. The HCP minimal preprocessing pipeline was used for the HCP

5 data set ${ }^{71}$. This pipeline includes artifact removal, motion correction, and registration to standard

6 space. After this pipeline, several standard preprocessing procedures, including linear detrend,

7 temporal filtering $(0.01-0.1 \mathrm{~Hz})$, regression of 12 motion parameters (HCP data; these include

8 first derivatives, given as Movement_Regressors_dt.txt) and mean time courses of the white

9 matter and CSF as well as the global signal, were applied to the fMRI data ${ }^{34}$. No spatial

10 smoothing was used in HCP MRI dataset preprocessing.

11 Functional connectivity calculation. All steps to construct resting-state FC networks were

12 identical to those used in the ANS dataset. Data from both LR and RL phase-encoding runs were

13 used to calculate connectivity matrices, respectively. The average of these two connectivity

14 matrices was used as the participants' connectivity matrices. Then a $201 \times 201 \mathrm{FC}$ matrix was

15 obtained for each participant.

\section{Numerosity CPM and numerosity network}

17 Numerosity $\boldsymbol{C P M}$. Numerosity CPM was established to predict individual differences in the

18 ANS acuity, using a leave-one-out (LOO) procedure ${ }^{35,36}$. One participant was left out for each

19 LOO iteration as the testing set, and the remaining participants were regarded as the training set.

20 The Spearman's rank correlation was calculated across participants between functional

21 connectivity of each edge in the FC matrix and Weber fraction. Consistent with previous

22 research ${ }^{35}$, for each LOO iteration, in the training set, the edges showing significant positive or

23 negative correlation with a $p$-value below a threshold $(p<0.01)$ across participants were included 
1 in a positive or negative network, respectively. The network strength of the training participant

2 was defined as the participant's summed strength of all FCs within the positive or negative

3 network. A general linear model (GLM) was fit to relate the summed strengths to the Weber

4 fraction for positive and negative networks, respectively. Then, in the testing set, the GLM was

5 used to obtain the predicted Weber fraction of the left-out participant from his/her summed FC

6 strength within the positive and negative network. After the LOO procedure was repeated for

7 each participant iteratively, we calculated the Pearson's correlation coefficient between the

8 observed and predicted Weber fractions across all participants to evaluate the predictive power.

9 The standard deviation (SD) method was applied to the Weber fraction to remove participants

10 with an outlier of the Weber fraction. The Weber fraction of each participant was within the

11 range of \pm three times SD around the mean value. No participant was removed from the ANS

12 primary dataset.

13 Permutation test. To confirm the reliability of prediction results, we fixed the predicted Weber

14 fraction and shuffled the observed Weber fraction across participants 10,000 times. We

15 calculated the Pearson's correlation between predicted and observed (randomly shuffled) scores.

16 The number of times that the correlation coefficients in the set of 10,000 permutation tests

17 outperformed the correlation coefficient from the numerosity CPM divided by 10,000 was

18 recorded as permutation test probability.

19 Numerosity network. Note that the positive or negative networks differed across iterations. To

20 obtain a unique numerosity network, we generated a final numerosity network by selecting the

21 overlapping edges across all LOO iterations' network of the numerosity CPM (i.e., the overall

22 network consisted of edges found in all LOO iterations). Then, the numerosity neuromarker was

23 defined as the summed FC strength within the final numerosity network in this study. We 
1 evaluated the predictive power of the numerosity neuromarker on several datasets, including an

2 independent ANS dataset and an HCP math/story dataset. Note that we used the numerosity

3 network to denote the final numerosity network.

\section{The predictive power of numerosity network}

$5 \quad$ The predictive power of numerosity network on ANS validating dataset. The ANS validating

6 dataset was used to confirm the reliable predictive power of the numerosity network to an

$7 \quad$ independent dataset with the same NC task. To evaluate the predictive power of the numerosity

8 network, we calculated the Pearson's correlation coefficients between the summed strength of the

9 numerosity network and the Weber fraction of the ANS validating dataset. The standard

10 deviation (SD) method (beyond the range of \pm three times SD around the mean value) was used

11 to exclude participants with outlier behavior performance from the correlation analysis. No

12 participant was excluded from the ANS validating dataset.

13 Permutation test. To confirm the significance of results on the ANS validating dataset, we fixed

14 the strength of the numerosity network and shuffled the observed Weber fraction 10,000 times,

15 and calculated the Pearson's correlation coefficients between them. Permutation test probability

16 was calculated by the number of times that correlation coefficients in a set of 10,000 permutation

17 tests outperformed the predictive power of the numerosity network, then divided by 10,000 .

18 The predictive power of numerosity network on HCP dataset. The HCP (math/story) dataset

19 was used to evaluate the predictive ability of the numerosity network to arithmetic skills and

20 language comprehension abilities. The predictive power of the numerosity network is evaluated

21 by Pearson's correlation coefficients between the strength of the numerosity network and the

22 behavioral performance of the HCP math/story dataset. Two participants (participant ID: 
1 172332, 217429) in the HCP math task and two participants (participant ID: 193239, 255639) in

2 the HCP story task were excluded because their performances were beyond the range of \pm three

3 times SD around the mean value. These four participants were excluded from the correlation

4 analysis. Permutation test procedures were the same as those for the ANS validating dataset.

5 Anatomical distribution of numerosity network. To determine the anatomical location of the

6 numerosity network, we grouped the 201 nodes into seven macroscale regions, including the

7 prefrontal (PFC), motor (Mot), insular (Ins), parietal (Par), temporal (Tem), occipital (Occ), and

8 limbic systems (Lim). We calculated the number of functional connections within or between all

9 the seven macroscale regions. To investigate the hub nodes in the numerosity network, we

10 ranked all nodes according to their degree centrality (DC; i.e., the number of direct functional

11 connections between a given node and the rest nodes within the numerosity network). . The

12 nodes and edges of the numerosity network were presented using BrainNet Viewer (V1.70,

13 www.nitrc.org/projects/bnv) ${ }^{72}$.

\section{References}

16 1. Feigenson L, Dehaene S, Spelke E. Core systems of number. Trends in Cognitive

$17 \quad$ Sciences 8, 307-314 (2004).

2. Barth H, Kanwisher N, Spelke E. The construction of large number representations in adults. Cognition 86, 201-221 (2003).

3. Pica P, Lemer C, Izard V, Dehaene S. Exact and Approximate Arithmetic in an Amazonian Indigene Group. Science (New York, NY) 306, 499-503 (2004). B11 (2000). 
5. Nieder A, Mller E. A parieto-frontal network for visual numerical information in the monkey. Proceedings of the National Academy of Sciences of the United States of America 101, 7457-7462 (2004).

6. Agrillo C, Parrish AE, Beran MJ. How Illusory Is the Solitaire Illusion? Assessing the Degree of Misperception of Numerosity in Adult Humans. Frontiers in Psychology 7, (2016).

7. Piffer L, Agrillo C, Hyde DC. Small and large number discrimination in guppies. Animal Cognition 15, 215-221 (2012).

8. Nasr K, Viswanathan P, Nieder A. Number detectors spontaneously emerge in a deep neural network designed for visual object recognition. Science advances $\mathbf{5}$, eaav7903 (2019).

9. Kim G, Jang J, Baek S, Song M, Paik S-B. Visual number sense in untrained deep neural networks. Science advances 7, eabd6127 (2021).

10. Zhou C, et al. Numerosity representation in a deep convolutional neural network. Journal of Pacific Rim Psychology 15, 18344909211012613 (2021).

11. Geary DC, Moore AM. Chapter 4 - Cognitive and brain systems underlying early mathematical development. In: Progress in Brain Research (eds Cappelletti M, Fias W). Elsevier (2016).

12. Gross HJ, Pahl M, Si A, Zhu H, Tautz J, Zhang S. Number-based visual generalisation in the honeybee. PloS one 4, e4263 (2009).

13. Piazza M. Neurocognitive start-up tools for symbolic number representations. Trends in Cognitive Sciences 14, 542-551 (2010).

14. Halberda J, Mazzocco MM, Feigenson L. Individual differences in non-verbal number acuity correlate with maths achievement. Nature 455, 665-668 (2008).

15. Nieder A. The neuronal code for number. Nature Reviews Neuroscience 17, 366-382 36 (2016).

16. Viswanathan $\mathrm{P}$, Nieder A. Neuronal correlates of a visual "sense of number" in primate parietal and prefrontal cortices. Proceedings of the National Academy of Sciences 110, 11187 (2013). 
17. Nieder A. Supramodal numerosity selectivity of neurons in primate prefrontal and posterior parietal cortices. Proceedings of the National Academy of Sciences 109, 11860 (2012).

18. Ramirez-Cardenas A, Nieder A. Working memory representation of empty sets in the primate parietal and prefrontal cortices. Cortex 114, 102-114 (2019).

19. Piazza M, Izard V, Pinel P, Le Bihan D, Dehaene S. Tuning Curves for Approximate Numerosity in the Human Intraparietal Sulcus. Neuron 44, 547-555 (2004).

20. Harvey BM, Ferri S, Orban GA. Comparing Parietal Quantity-Processing Mechanisms between Humans and Macaques. Trends in Cognitive Sciences 21, 779-793 (2017).

21. Hawes Z, Sokolowski HM, Ononye CB, Ansari D. Neural underpinnings of numerical and spatial cognition: An fMRI meta-analysis of brain regions associated with symbolic number, arithmetic, and mental rotation. Neuroscience \& Biobehavioral Reviews 103, 316-336 (2019).

22. Nieder A. Evolution of cognitive and neural solutions enabling numerosity judgements: lessons from primates and corvids. Philosophical transactions of the Royal Society of London Series B, Biological sciences 373, (2017).

23. Harvey BM. Quantity Cognition: Numbers, Numerosity, Zero and Mathematics. Current Biology 26, R419-R421 (2016).

24. Fornaciai M, Brannon EM, Woldorff MG, Park J. Numerosity processing in early visual cortex. NeuroImage 157, 429-438 (2017).

25. Park J, Dewind N, Woldorff M, Brannon E. Rapid and Direct Encoding of Numerosity in the Visual Stream. Cerebral cortex (New York, NY : 1991) 26, (2015).

26. Lasne G, Piazza M, Dehaene S, Kleinschmidt A, Eger E. Discriminability of numerosityevoked fMRI activity patterns in human intra-parietal cortex reflects behavioral numerical acuity. Cortex 114, 90-101 (2019).

27. Kutter EF, Bostroem J, Elger CE, Mormann F, Nieder A. Single Neurons in the Human Brain Encode Numbers. Neuron 100, 753-761.e754 (2018). 


\section{Dehaene S, Piazza M, Pinel P, Cohen L. THREE PARIETAL CIRCUITS FOR} NUMBER PROCESSING. Cognitive Neuropsychology 20, 487-506 (2003).

29. Dehaene S, Cohen L. Cerebral Pathways for Calculation: Double Dissociation between Rote Verbal and Quantitative Knowledge of Arithmetic. Cortex 33, 219-250 (1997).

30. Biswal B, Yetkin FZ, Haughton VM, Hyde JS. Functional connectivity in the motor cortex of resting human brain using echo-planar MRI. Magnetic resonance in medicine 34, 537-541 (1995).

31. Goense J, Whittingstall K, Logothetis N. Neural and BOLD responses across the brain. Wiley Interdisciplinary Reviews: Cognitive Science 3, 75-86 (2012).

32. Smith SM, et al. Functional connectomics from resting-state fMRI. Trends in Cognitive Sciences 17, 666-682 (2013).

33. Shen $\mathrm{X}$, et al. Using connectome-based predictive modeling to predict individual behavior from brain connectivity. Nature Protocols 12, 506-518 (2017).

34. Emily S, et al. Functional connectome fingerprinting: identifying individuals using patterns of brain connectivity. Nature neuroscience, (2015).

35. Rosenberg MD, et al. A neuromarker of sustained attention from whole-brain functional connectivity. Nature Neuroscience 19, 165-171 (2016).

39. Lukowski SL, et al. Approximate number sense shares etiological overlap with mathematics and general cognitive ability. Intelligence 65, 67-74 (2017).

40. Townsend J, Ashby FG. Methods of modeling capacity in simple processing systems. Cogn Theory 3, 200-239 (1978). 
41. Shen X, Tokoglu F, Papademetris X, Constable RT. Groupwise whole-brain parcellation from resting-state fMRI data for network node identification. NeuroImage 82, 403-415 (2013).

42. Steiger, James H. Tests for comparing elements of a correlation matrix. Psychological Bulletin 87, 245-251 (1980).

43. DeWind NK, Park J, Woldorff MG, Brannon EM. Numerical encoding in early visual cortex. Cortex 114, 76-89 (2019).

44. Klein E, Willmes K, Bieck SM, Bloechle J, Moeller K. White matter neuro-plasticity in mental arithmetic: Changes in hippocampal connectivity following arithmetic drill training. Cortex 114, 115-123 (2019).

45. Anobile G, Arrighi R, Castaldi E, Burr DC. A Sensorimotor Numerosity System. Trends in Cognitive Sciences 25, 24-36 (2021).

46. Wang X, Jiao D, Zhang X, Lin X. Altered degree centrality in childhood absence epilepsy: A resting-state fMRI study. Journal of the Neurological Sciences 373, 274-279 (2017).

47. Starr A, Libertus ME, Brannon EM. Number sense in infancy predicts mathematical abilities in childhood. Proceedings of the National Academy of Sciences 110, 18116 (2013).

48. Amalric M, Dehaene S. A distinct cortical network for mathematical knowledge in the human brain. NeuroImage 189, 19-31 (2019).

49. Amalric M, Dehaene S. Origins of the brain networks for advanced mathematics in expert mathematicians. Proc Natl Acad Sci U S A 113, 4909-4917 (2016).

50. Amalric M, Dehaene S. Cortical circuits for mathematical knowledge: evidence for a major subdivision within the brain's semantic networks. Philosophical transactions of the Royal Society of London Series B, Biological sciences 373, (2017).

51. Voets NL, et al. Functional and structural changes in the memory network associated with left temporal lobe epilepsy. Human brain mapping 30, 4070-4081 (2009). 
52. Leibovich T, Katzin N, Harel M, Henik A. From sense of numbe' to sense of magnitud... : The role of continuous magnitudes in numerical cognition. Behavioral and Brain Sciences 40, (2016).

53. Piazza M, De Feo V, Panzeri S, Dehaene S. Learning to focus on number. Cognition 181, 35-45 (2018).

54. Starr A, DeWind NK, Brannon EM. The contributions of numerical acuity and nonnumerical stimulus features to the development of the number sense and symbolic math achievement. Cognition 168, 222-233 (2017).

55. Wilkey ED, Price GR. Attention to number: The convergence of numerical magnitude processing, attention, and mathematics in the inferior frontal gyrus. Human brain mapping 40, 928-943 (2019).

56. Castaldi E, Mirassou A, Dehaene S, Piazza M, Eger E. Asymmetrical interference between number and item size perception provides evidence for a domain specific impairment in dyscalculia. PloS one 13, e0209256 (2018).

57. Park J, DeWind NK, Woldorff MG, Brannon EM. Rapid and Direct Encoding of Numerosity in the Visual Stream. Cereb Cortex 26, 748-763 (2016).

58. Sawamura H, Shima K, Tanji J. Numerical representation for action in the parietal cortex of the monkey. Nature 415, 918-922 (2002).

59. Westendorp M, Hartman E, Houwen S, Smith J, Visscher C. The relationship between gross motor skills and academic achievement in children with learning disabilities. Research in Developmental Disabilities 32, 2773-2779 (2011).

60. Piazza M, Mechelli A, Price CJ, Butterworth B. Exact and approximate judgements of visual and auditory numerosity: An fMRI study. Brain Research 1106, 177-188 (2006).

61. Piazza M, Pinel P, Le Bihan D, Dehaene S. A Magnitude Code Common to Numerosities and Number Symbols in Human Intraparietal Cortex. Neuron 53, 293-305 (2007).

62. Arsalidou M, Taylor MJ. Is $2+2=4$ ? Meta-analyses of brain areas needed for numbers and calculations. NeuroImage 54, 2382-2393 (2011).

63. Nieder A, Freedman DJ, Miller EK. Representation of the quantity of visual items in the 
64. Dehaene S, Molko N, Cohen L, Wilson AJ. Arithmetic and the brain. Current Opinion in Neurobiology 14, 218-224 (2004).

65. Piazza M, Pica P, Izard V, Spelke ES, Dehaene S. Education enhances the acuity of the nonverbal approximate number system. Psychological science 24, 1037-1043 (2013).

66. Watson AB, Pelli DG. Quest: A Bayesian adaptive psychometric method. Perception \& Psychophysics 33, 113-120 (1983).

67. Sims J, Pelli D. The Ideal Psychometric Procedure. (1987).

68. Baldassi S, Burr DC. Feature-based integration of orientation signals in visual search. Vision Research 40, 1293-1300 (2000).

69. Shen X, Papademetris X, Constable RT. Graph-theory based parcellation of functional subunits in the brain from resting-state fMRI data. NeuroImage 50, 1027-1035 (2010).

70. Binder JR, et al. Mapping anterior temporal lobe language areas with fMRI: A

71. Glasser MF, et al. The minimal preprocessing pipelines for the Human Connectome Project. NeuroImage 80, 105-124 (2013).

72. Xia M, Wang J, He Y. BrainNet Viewer: a network visualization tool for human brain connectomics. PloS one 8, e68910 (2013).

\section{Acknowledgments}

This work was supported by the National Key R\&D Program of China (2019YFA0709503),

31 Foundation of China grant $(31671133,61971289)$, Shenzhen Science and Technology Research

32 Funding Program (JCYJ20170412164413575, JCYJ20170412111316339), Fundamental

33 Research Funds for the Central Universities, and Shenzhen-Hong Kong Institute of Brain

34 Science-Shenzhen Fundamental Research Institutions.

\section{Competing interests}


1 The authors declare no competing interests.

3 Data availability

4 Each participant's functional matrix and behavior performance from the ANS dataset and HCP

5 math/story dataset are available from the authors upon request.

6

7 Code availability

8 Matlab scripts were written to identify numerosity neuromarker, make predictions from novel

9 individuals' FC matrices, and generalize the numerosity neuromarker to ANS validating dataset

10 and HCP math/story dataset. These codes are available online from

11 https://github.com/Dzhang1989z/Numerosity-CPM. 


\section{$1 \quad$ Figures}

A
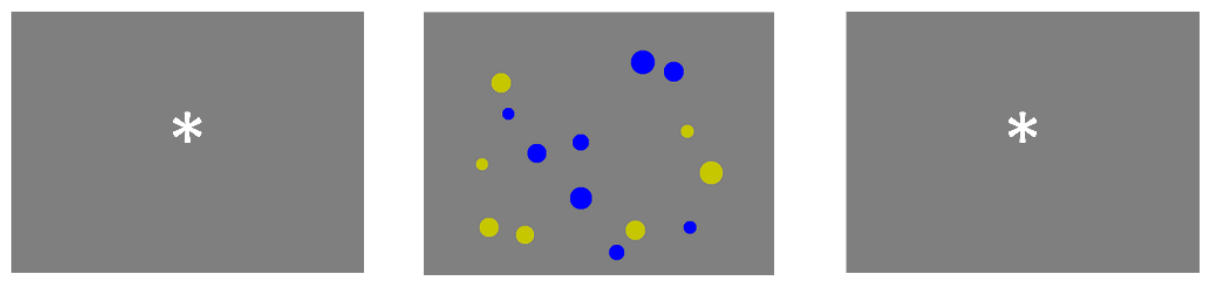

Fixation (300 ms)

$750 \mathrm{~ms}$

Until response

B

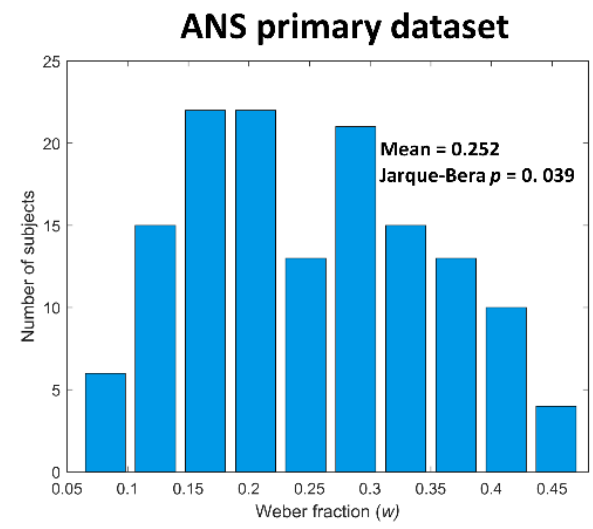

D
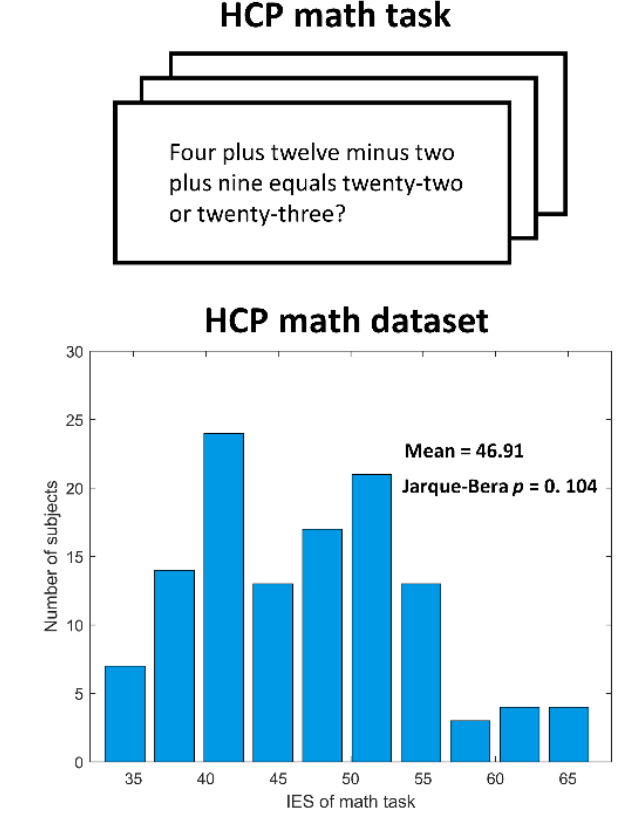

C

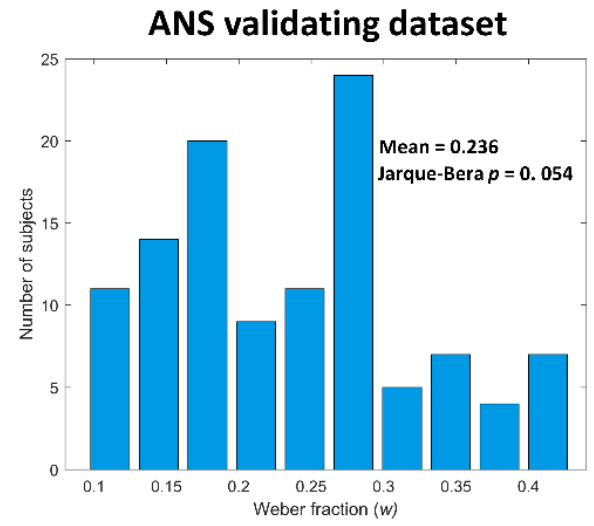

$\mathbf{E}$

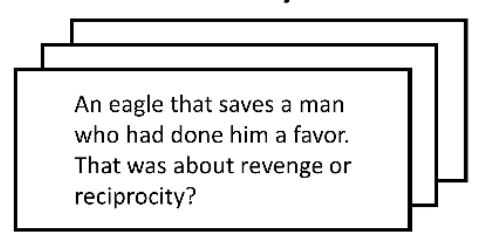

HCP story dataset

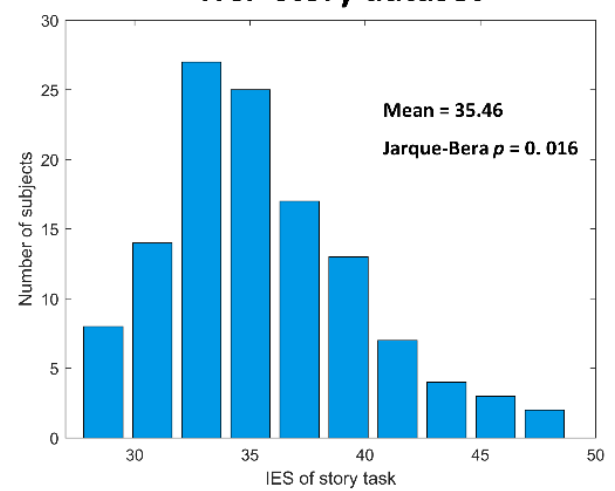

Fig 1. Experimental task and group performance for the ANS dataset and HCP story/math dataset. (A) The paradigm for the NC task. (B) Histogram of weber fraction w, the ANS acuity, for the ANS primary dataset ( $\mathrm{n}=141)$, as determined by a psychophysical model for each participant. (C) Histogram of weber fraction $w$ for the ANS validating dataset (n=112). (D/E) Experimental task and histogram of the IES performance for the HCP math/story dataset $(\mathrm{n}=120)$. 

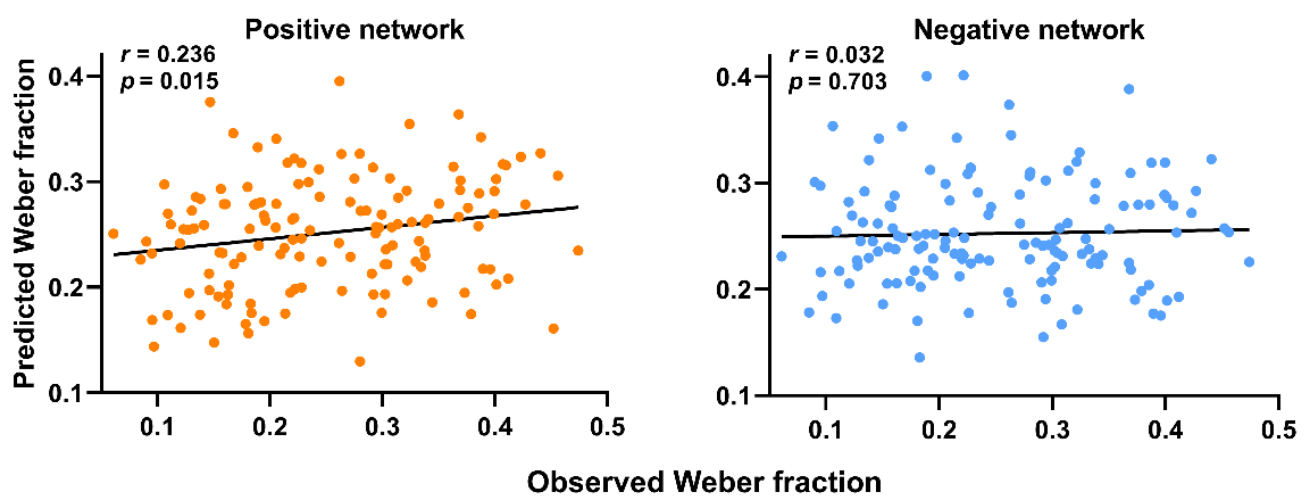

2 Fig 2. CPMs predict Weber fraction. Scatter plots show correlations between observed Weber fraction and predictions by positive (left) and negative (right) networks. Network models were iteratively trained on behavior and MRI data from n-1 participants in the ANS primary dataset and tested on the left-out participant. The $r$ and $p$-value above each plot show the Pearson's correlation coefficient between observed and predicted Weber fraction, and corresponding significance level. 
A

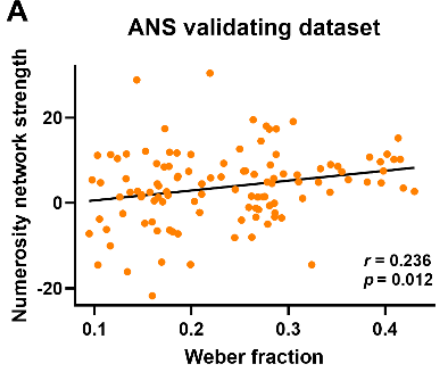

B

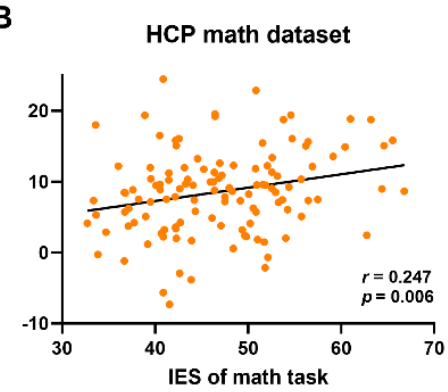

C

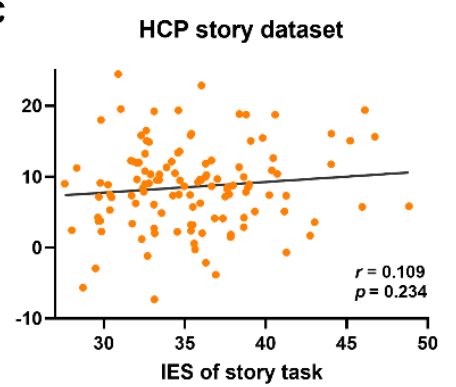

2 Fig 3. The predictive power of numerosity neuromarker on several independent datasets. Pearson's correlations between

3 network strength of numerosity network and behavior performance in the ANS validating dataset (A), HCP math dataset (B), and

4 HCP story dataset (C) were calculated to assess the predictive power of numerosity neuromarker. The meaning of $r$ and $p$ shows

5 the Pearson's correlation coefficient between numerosity network strength and each behavior performance, and corresponding

6 significance level, respectively. 
A
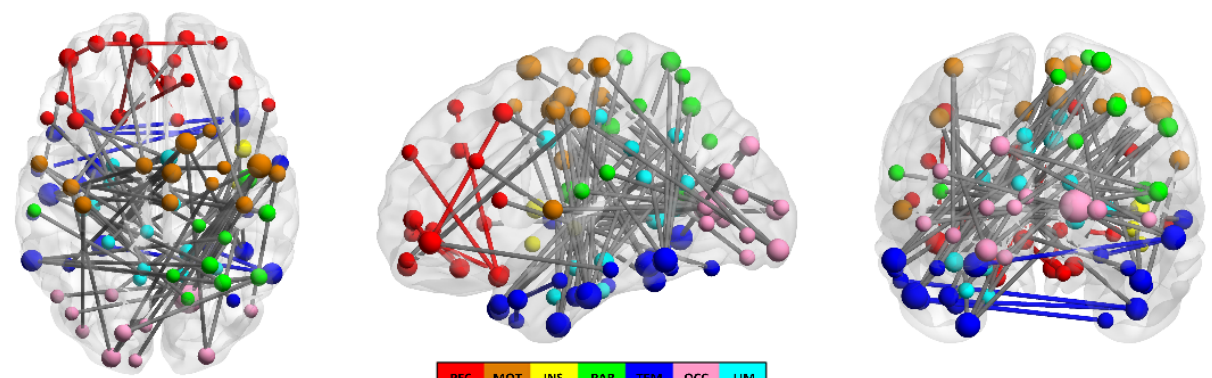

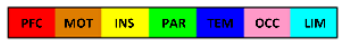

B

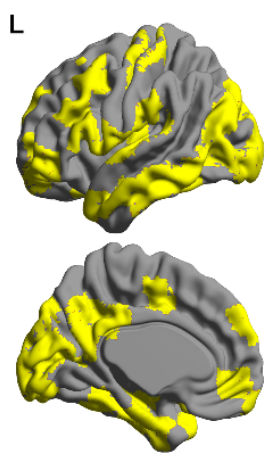

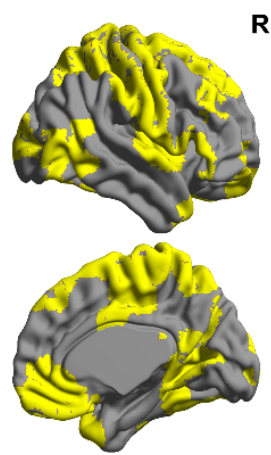

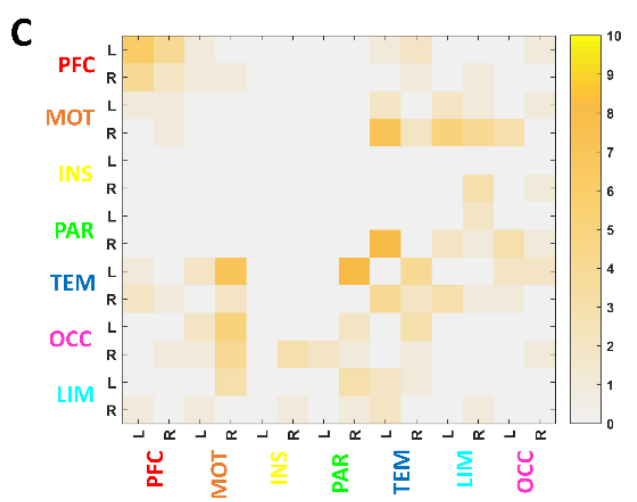

Fig 4. Anatomical locations of the numerosity network. (A) A 3d view of numerosity network edges and nodes on a glass brain. Nodes in the cerebellum, subcortical, and brainstem were not included in our analysis. Hence, Macroscale regions include the prefrontal cortex (PFC), the motor cortex (Mot), insula (Ins), parietal (Par), temporal (Tem), occipital (Occ), limbic (including the cingulate cortex, amygdala, and hippocampus; Lim). The larger size of a node indicates that the node gets a larger degree centrality (DC) value. (B) Cortical areas of the numerosity network. (C) All edges are grouped by macroscale region and hemisphere $(\mathrm{L}=$ left, $\mathrm{R}=$ right). Colorbar values indicate the number of edges within or between the macroscale regions. 

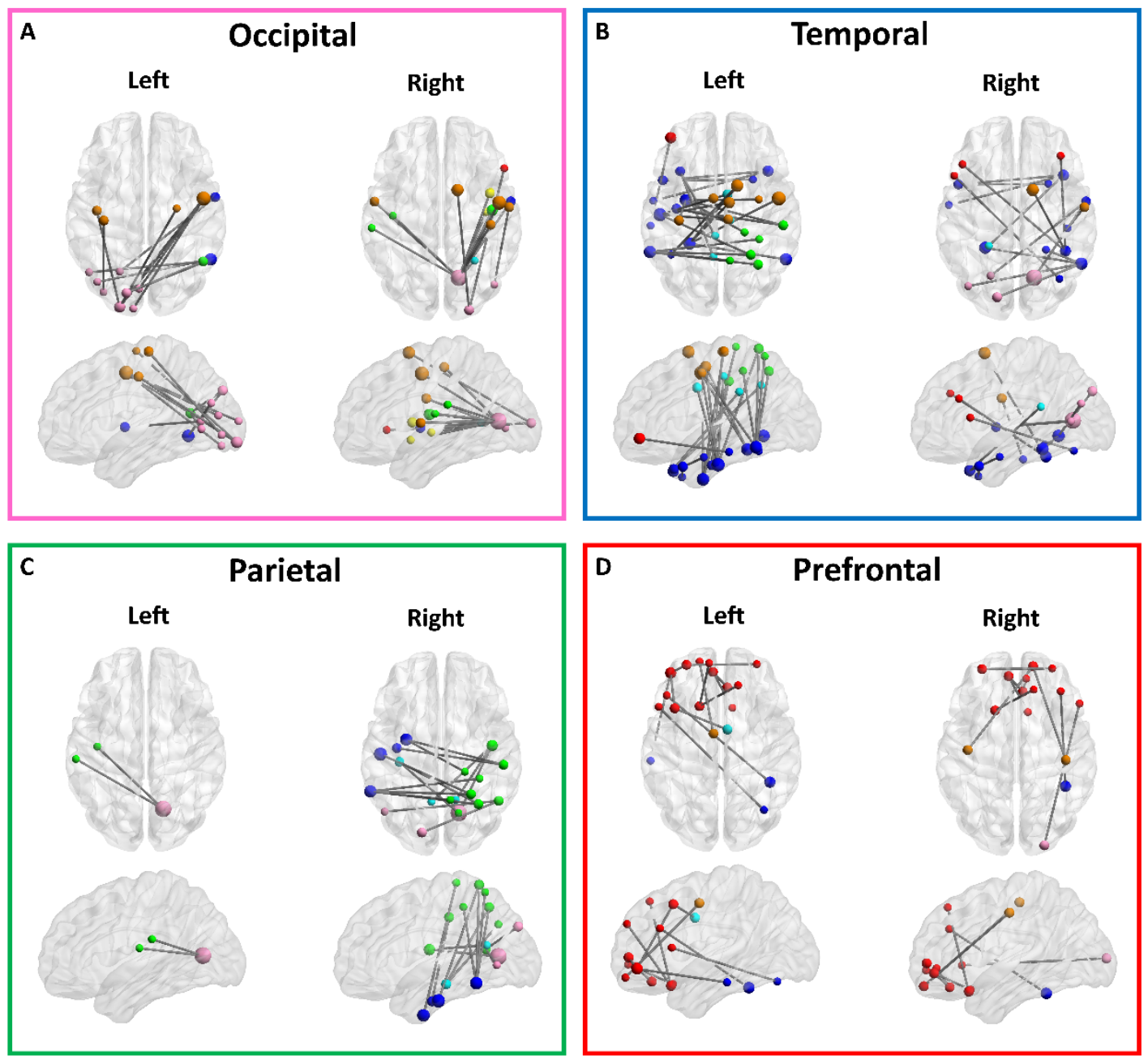

2 Fig 5. Functional connections in the numerosity network in each of four macroscale regions. (A) occipital, (B) temporal, (C) parietal, and (D) prefrontal regions. Dot color indicates macroscale region as in Fig. 4. 


\section{$1 \quad$ Tables}

2 Table 1. Summary of behavioral data. Jarque-Bera tests indicated significant departures from normality, supporting the use of 3 Spearman's rank correlations. Because we sought to identify any potential departures from normality, no correction for multiple 4 comparisons was applied across these tests. All p-values are based on two-tailed tests.

\begin{tabular}{|l|l|l|l|l|l|l|}
\hline Task & Metric & Participant \# & Mean & SD & Range & Jarque-Bera(p) \\
\hline ANS primary dataset & Weber fraction & 141 & 0.252 & 0.099 & {$[0.061,0.474]$} & 0.039 \\
\hline ANS validating dataset & Weber fraction & 112 & 0.236 & 0.088 & {$[0.094,0.430]$} & 0.054 \\
\hline HCP math & Inverse efficiency score & 120 & 46.910 & 7.713 & {$[32.72,66.77]$} & 0.104 \\
\hline HCP story & Inverse efficiency score & 120 & 35.461 & 4.290 & {$[27.58,48.84]$} & 0.016 \\
\hline
\end{tabular}


1 Table 2. Information of the hub nodes $(\mathrm{DC}>1)$ in the numerosity network.

\begin{tabular}{|c|c|c|c|c|c|c|}
\hline No. & DC & Node name & $\mathbf{L} / \mathbf{R}$ & Macroscale region & BA & MNI \\
\hline 1 & 11 & Primary visual cortex & $\mathrm{R}$ & Occipital & 17 & $(14.6,-68.3,8.3)$ \\
\hline 2 & 6 & Premotor cortex & $\mathrm{R}$ & Motor & 6 & $(49.2,-4.6,48.1)$ \\
\hline 3 & 5 & Inferior temporal gyrus & $\mathrm{L}$ & Temporal & 20 & $(-51.8,-18.2,-28.8)$ \\
\hline 4 & 4 & Fusiform gyrus & $\mathrm{L}$ & Temporal & 37 & $(-60.3,-50.0,-14.0)$ \\
\hline 5 & 4 & Premotor cortex & $\mathrm{R}$ & Motor & 6 & $(13.7,6.3,65.4)$ \\
\hline 6 & 4 & Fusiform gyrus & $\mathrm{L}$ & Temporal & 37 & $(-26.7,-42.7,-16.1)$ \\
\hline 7 & 4 & Fusiform gyrus & $\mathrm{R}$ & Temporal & 37 & $(55.2,-56.3,-4.8)$ \\
\hline 8 & 4 & Parahippocampal cortex & $\mathrm{L}$ & Temporal & 36 & $(-30.0,-5.8,-40.9)$ \\
\hline 9 & 3 & Fusiform gyrus & $\mathrm{R}$ & Temporal & 37 & $(41.6,-45.7,-22.6)$ \\
\hline 10 & 3 & Frontopolar area & $\mathrm{L}$ & Prefrontal & 10 & $(-42.7,47.3,-6.9)$ \\
\hline 11 & 3 & Temporopolar area & $\mathrm{R}$ & Temporal & 38 & $(40.0,18.9,-34.2)$ \\
\hline 12 & 3 & Primary sensory cortex & $\mathrm{R}$ & Parietal & 1 & $(43.3,-10.8,13.9)$ \\
\hline 13 & 3 & Supplementary motor area & $\mathrm{R}$ & Motor & 6 & $(7.0,-8.1,52.9)$ \\
\hline 14 & 3 & Visual association cortex & $\mathrm{L}$ & Occipital & 18 & $(-22.3,-96,6,-10.1)$ \\
\hline 15 & 2 & Orbitofrontal cortex & $\mathrm{L}$ & Prefrontal & 11 & $(-18.2,19.0,-21)$ \\
\hline 16 & 2 & Ventral anterior cingulate gyrus & $\mathrm{R}$ & Limbic & 24 & $(5.3,-1.0,35.6)$ \\
\hline 17 & 2 & Angular gyrus & $\mathrm{R}$ & Parietal & 39 & $(48.9,-58.1,14.4)$ \\
\hline 18 & 2 & Frontopolar area & $\mathrm{L}$ & Prefrontal & 10 & $(-6.9,48.3,-5.7)$ \\
\hline 19 & 2 & Orbitofrontal area & $\mathrm{R}$ & Prefrontal & 11 & $(5.1,34.9,-17.4)$ \\
\hline 20 & 2 & Primary sensory cortex & $\mathrm{R}$ & Motor & 1 & $(42.0,-23.4,53.4)$ \\
\hline 21 & 2 & Paracentral lobule & $\mathrm{R}$ & Motor & 6 & $(6.0,-22.3,65.6)$ \\
\hline 22 & 2 & Primary motor cortex & $\mathrm{R}$ & Motor & 4 & $(57.8,-8.3,27.3)$ \\
\hline 23 & 2 & Inferior temporal gyrus & $\mathrm{L}$ & Temporal & 20 & $(-49.3,-4.7,-37.4)$ \\
\hline 24 & 2 & Visual association cortex & $\mathrm{R}$ & Occipital & 18 & $(23.7,-96.0,6.5)$ \\
\hline 25 & 2 & Middle frontal gyrus & $\mathrm{L}$ & Prefrontal & 8 & $(-39.3,17.2,46.7)$ \\
\hline 26 & 2 & Inferior parietal gyrus & $\mathrm{R}$ & Parietal & 40 & $(52.8,-27.2,40.9)$ \\
\hline 27 & 2 & Orbitofrontal cortex & $\mathrm{R}$ & Prefrontal & 11 & $(13.9,56.8,-16.6)$ \\
\hline
\end{tabular}

BA, Brodmann area; DC, degree centrality; L, left; R, right. 


\section{Supplementary information}

\section{Fine spatial distribution of numerosity network}

The ROIs to which the occipital region is connected in numerosity network are visualized in Fig. 5A. The occipital (primary visual and visual association) nodes are mainly connected to temporal, parietal and motor regions. To be specific, the left occipital regions connect to right premotor cortex, left primary motor cortex, left primary sensory cortex, right angular gyrus, bilateral primary sensory cortex, right superior temporal cortex and right fusiform gyrus; the right occipital regions connect to right primary motor cortex, bilateral premotor cortex, bilateral primary sensory cortex, left supramarginal gyrus (SMG) and right superior temporal cortex.

The temporal regions played an outsize contribution in the numerosity network (Fig. 5B). The left temporal and right temporal region show sort of dissimilar connection patterns. The connection between left temporal (temporopolar area, medial temporal gyrus, inferior temporal gyrus and fusiform gyrus) nodes and right motor, right parietal regions is especially dense, while no connections between right temporal and parietal cortex was found. To be specific, the left temporal nodes mainly connected to several pre-motor nodes, right parietal sensory areas (located near the intraparietal sulcus, IPS) and bilateral ventral/dorsal posterior cingulate cortex (PCC); the right temporal nodes connected to a part of bilateral prefrontal cortex (PFC), right motor cortex, left temporal cortex and bilateral occipital cortex.

The ROIs connected to the parietal region are visualized in Fig. 5C. In the parietal region, most connections locate between right parietal sensory nodes and left temporal cortex, bilateral occipital cortex, as mentioned previously. The left parietal regions (primary sensory and SMG) merely connect with the primary visual area. The right parietal regions connect with the left inferior temporal gyrus (ITG), left fusiform gyrus, left parahippocampal gyrus (PPA), right primary visual cortex and left visual association cortex.

The right motor regions got more dense connections than the left motor regions (supplementary Fig. 2A). The motor (primary motor/sensory and premotor) nodes are mainly connected with occipital and temporal cortex. To be specific, the left motor regions connect with left ITG, left PPA, right primary visual and left visual association nodes; the right motor regions primarily connect with bilateral ITG, bilateral fusiform gyrus, left PPA, bilateral primary visual and visual association nodes.

The prefrontal cortex shows most connections located within prefrontal lobe (Fig. 5D). Besides the intra-lobe connections, the left prefrontal regions primarily connect with left premotor cortex, right fusiform gyrus and left medial temporal gyrus (MTG); the right prefrontal region connects to right primary sensory/motor cortex, right fusiform gyrus, right visual association area.

The limbic cortex shows connections with all other six macroscale regions excepting insula (supplementary Fig. 2B). Limbic cortex connects more densely with motor and parietal cortex than other cortices. 

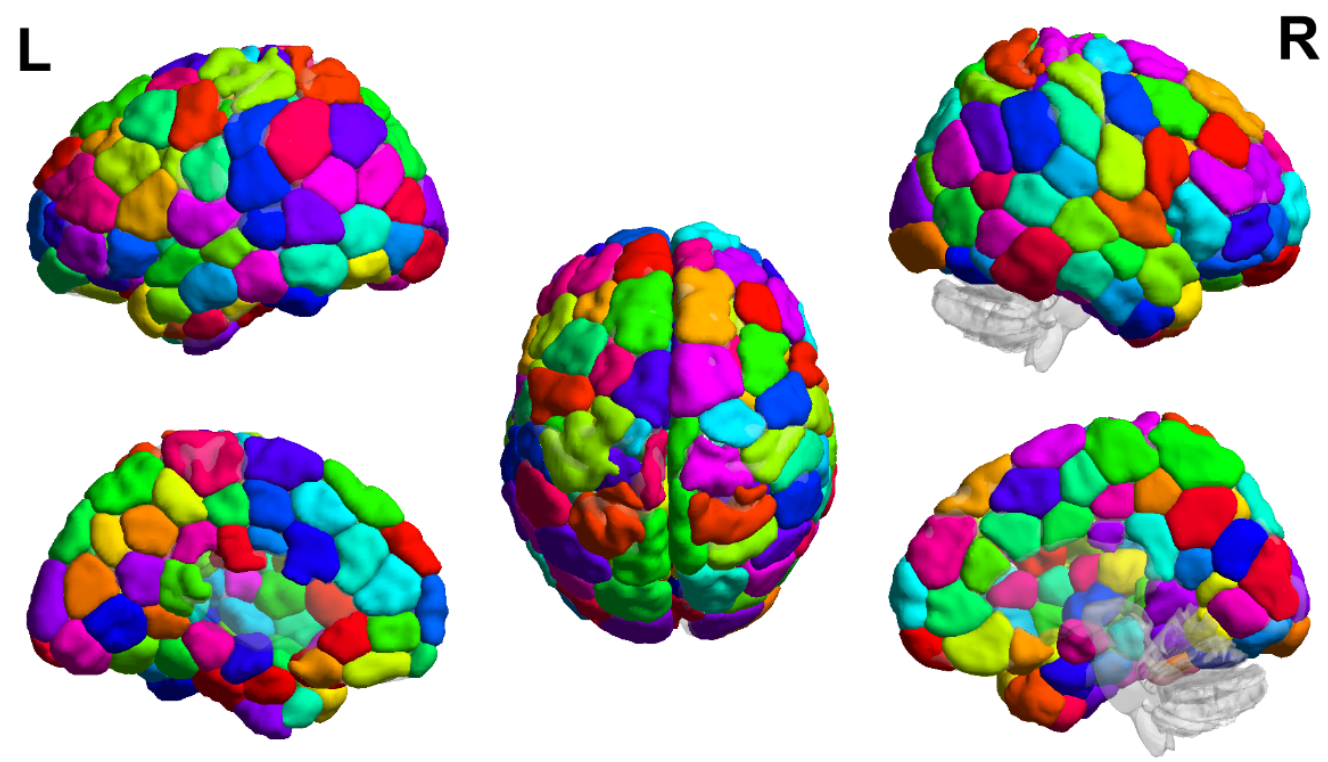

Supplementary Fig. 1. The 201-region functional parcellation used to define network nodes in primary NC dataset. Colored nodes were included in the FC matrix calculation. All nodes (67 nodes) in the cerebellum, brainstem, and subcortex were not included in our analysis. 

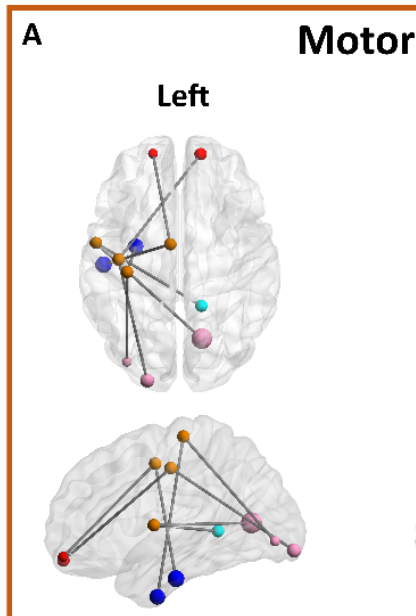

B Right
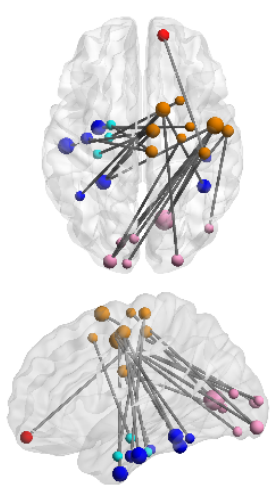

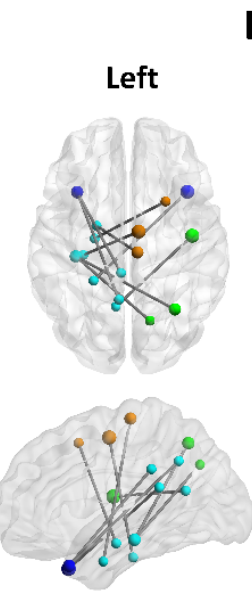

Right
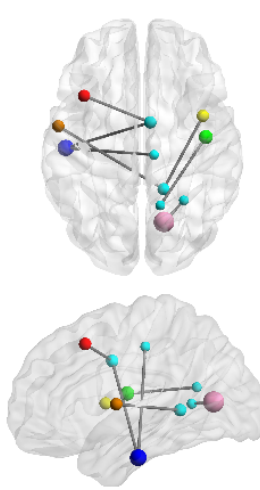

Supplementary Fig. 2. All connections in the numerosity network that include nodes in (A) motor and (B) limbic system. Dot color indicates macroscale region as in Fig. 4. 